\title{
The importance of distinguishing Mycoplasma pneumoniae-induced rash and mucositis (MIRM) and Stevens-Johnson syndrome in a clinical setting
}

\author{
Roman Pillay ${ }^{1}$ and Suran L Fernando ${ }^{2 *}$ \\ ${ }^{1}$ Department of Clinical Immunology and Allergy, Royal North Shore Hospital, Australia \\ ${ }^{2}$ Immunology Laboratory, NSW Health Pathology, Department of Clinical Immunology and Allergy, Royal North Shore Hospital and University of Sydney, Australia
}

\begin{abstract}
Mycoplasma pneumoniae-induced rash and mucositis (MIRM) is a distinct entity from Stevens-Johnson syndrome/toxic epidermal necrolysis and erythema multiforme. As antibiotics may be prescribed in the context of MIRM, it is critical to differentiate drug hypersensitivity from Mycoplasma pneumoniae-sequelae to avoid incorrect drug allergy labeling. Patients may require evaluation by an immunologist for removal of a drug allergy label in cases of MIRM through clinical evaluation with or without a protocolised drug challenge.
\end{abstract}

\section{Introduction}

Mycoplasma pneumoniae, a leading cause of community-acquired pneumonia, causes various extrapulmonary manifestations, including rash and mucositis (MIRM). These have been reported in up to $25 \%$ of paediatric patients (mean age 12), with adult infections occurring less commonly [1,2]. MIRM was coined in 2015 to differentiate it from Stevens-Johnson syndrome/toxic epidermal necrolysis (SJS/ TEN) although historically it has been referred to as Fuchs syndrome, an atypical variant of SJS characterised by mucositis in the absence of cutaneous lesions [3]. It was named to also distinguish it from erythema multiforme (EM) in which multiple mucosal surface involvement is not present [4]. We present a case of MIRM in an adult that was initially diagnosed as a SJS secondary to amoxicillin resulting in a penicillin allergy that required subsequent delabelling by the immunology service.

\section{Main Text}

A 24-year-old male presented to hospital with oral mucositis, conjunctivitis and urethritis, in the absence of rash and a background of a respiratory tract infection. His symptoms began 14 days prior with a prodrome comprising fevers, chills, myalgia and nausea. On day 4 , he developed a cough with gritty sensation affecting both eyes. On day 5 , he was prescribed amoxicillin by his local medical officer presumptively for a respiratory tract infection, and topical chloramphenicol eye drops for bilateral conjunctivitis. The following day, he attended his local emergency department as his cough became productive of mucopurulent sputum and his ocular symptoms worsened with a purulent discharge (Figure 1).

On examination he was febrile $\left(39.4^{\circ} \mathrm{C}\right)$, tachycardic $(117 / \mathrm{min})$ and had reduced breath sounds in the right middle zone of his chest. Laboratory investigations showed an elevated C-reactive protein 65 $\mathrm{mg} / \mathrm{L}(<5)$. His total white cell count $\left(5.0 \times 10^{9}\right.$ cells/L) and differential was normal. His blood cultures were negative. His chest $\mathrm{x}$-ray revealed right middle lobe consolidation (Figure 2). He was changed from amoxicillin to ampicillin and doxycycline, and a single dose of ceftriaxone for treatment of community acquired pneumonia.

On day 7 , he developed sloughing of his oral mucosa with odynophagia (Figure 3). On day 9, he developed painful urination and purulent discharge secondary to urethritis. His urine culture did not isolate a bacterial pathogen and his PCR for N. gonorrhoea and C. trachomatis was negative. His conjunctivitis remained unchanged and did not progress to involve the cornea, and swabs of his ocular discharge did not detect viral or bacterial pathogens.

However, a nasopharyngeal swab was positive for M. pneumoniae in the context of positive M. pneumoniae serology (total antibody level 160) and negative sputum culture and respiratory virus PCR. His antibiotic therapy was subsequently changed to roxithromycin. $\mathrm{He}$ was transferred to a tertiary centre for ongoing multidisciplinary team management comprising of ENT, ophthalmology and urology reviews. Chloramphenicol and lubricating eye drops were continued for conjunctivitis. ENT review demonstrated mild pharyngeal involvement and he was treated with sodium bicarbonate mouthwash, and topical lignocaine anaesthetic. No intervention was required for his urethritis.

His desquamating oral mucositis, urethritis and conjunctivitis as well as his clinical and radiological features of his Mycoplasma pneumoniae completely resolved over 4 weeks. He never developed a skin rash during the course of his illness.

Immunology review determined that a delayed drug hypersensitivity which is the pathomechanism for a SJS variant such as mucositis was unlikely as he developed conjunctivitis prior to the

${ }^{\star}$ Correspondence to: Suran L Fernando, Immunology Laboratory, NSW Health Pathology, Department of Clinical Immunology and Allergy, Royal North Shore Hospital and University of Sydney, Australia, Tel: Ph : 029926 4108; Fax : 02 09463 1058; E-mail: Suran.fernando@health.nsw.gov.au

Received: April 21, 2020; Accepted: May 07, 2020; Published: May 09, 2020 


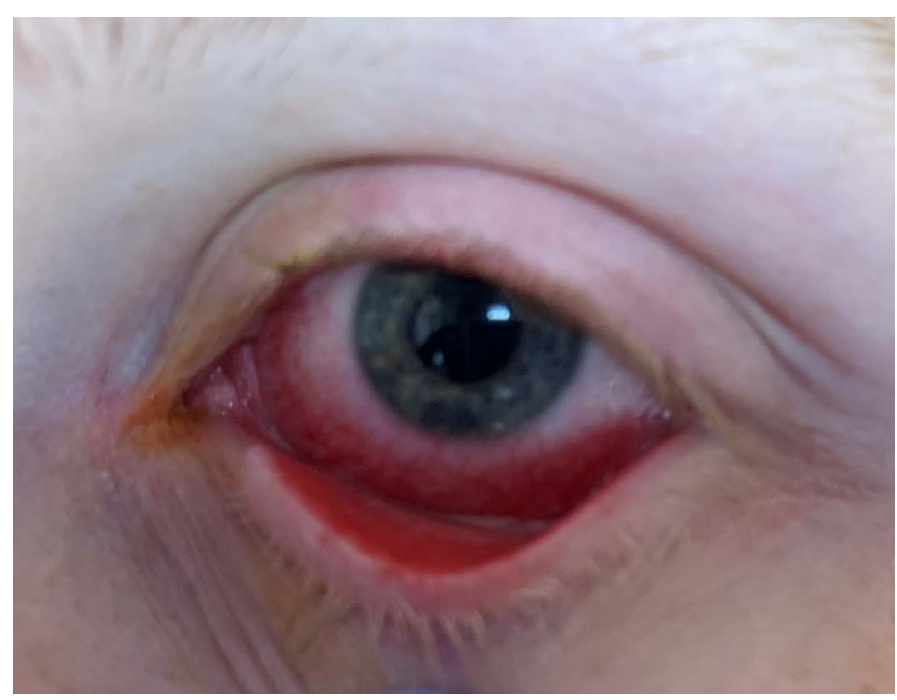

Figure 1. Conjunctivitis

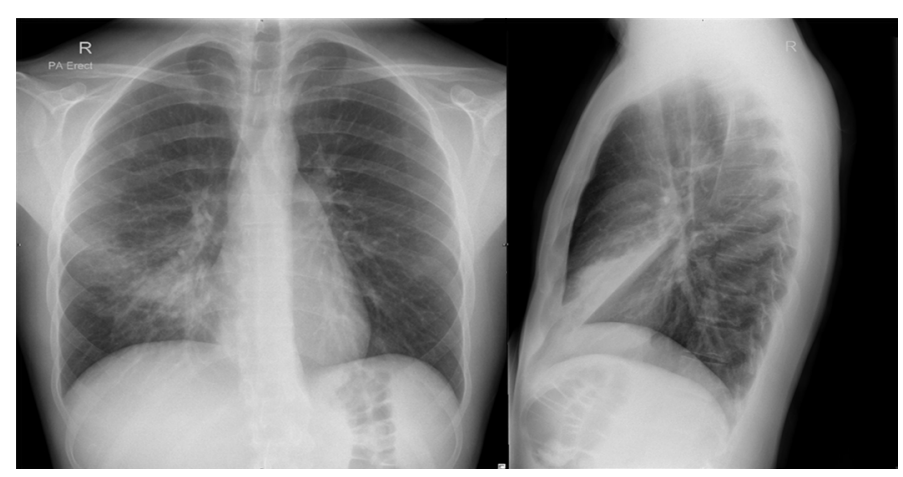

Figure 2. Chest x-ray showing right middle lobe consolidation from M. pneumoniae infection

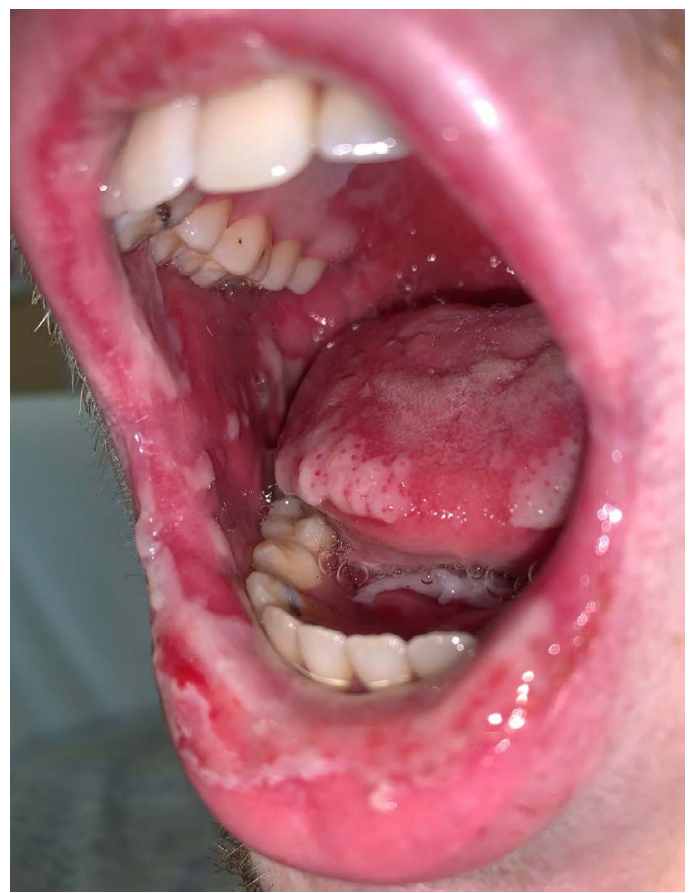

Figure 3. Oral mucositis with desquamation of the buccal mucosa, soft palate, tongue and lips ingestion of amoxicillin and the time from amoxicillin to the onset oral mucositis and urethritis was 3 days. Nevertheless, he was labelled as having a penicillin drug allergy in the electronic medical record and was subsequently successfully delabelled by the Immunology Service according to the Australasian Society for Clinical Immunology and Allergy Society Guidelines with a 3 day oral challenge to amoxicillin.

\section{Discussion}

MIRM should be suspected in a child or young patient presenting with a mucosal or mucocutaneous eruption and a history of prodromal symptoms preceding the eruption by approximately one week [5]. The diagnostic criteria are mucocutaneous eruption involving less than $10 \%$ of the body surface area, involvement of at least 2 mucosal surfaces, sparsely scattered targetoid or vesiculobullous lesions with some patients having no cutaneous involvement (MIRM sine rash) and clinical and laboratory evidence of M. pneumoniae infection [5].

There are no pathognomonic histopathologic determinants in MIRM and there are many overlapping features with both SJS and EM such as apoptotic keratinocytes, perivascular dermal infiltrate, and full-thickness epidermal necrosis and detachment. M. pneumoniae has not been detected in biopsy samples [6]. The pathogenesis remains unclear but there is some evidence of molecular mimicry in the antibody response to M. pneumoniae and P1-adhesion molecules and keratinocyte antigens resulting in immune complex formation, complement activation and mucocutaenous tissue damage [7]. No genetic susceptibility alleles have been detected as in a number of severe cutaneous adverse drug reactions. It remains to be determined whether important mediators of apoptosis in SJS/TEN such as granulysin and IL-15 are also detected in MIRM, or whether other mediators play a prominent role since the mediators in SJS/TEN correlate with disease severity, and MIRM has a benign course.

The major differential diagnosis of MIRM is drug hypersensitivity. An accurate history often differentiates between drugs and infection as the cause of such reactions. Typically, drug-induced SJS occurs between 4 days to 4 weeks after exposure and penicillins are rarely attributed to these reactions [8]. Further, clinical evaluation would have revealed no epidermal detachment, a hallmark of SJS. Nevertheless, a penicillin allergy label was stipulated in the patient's electronic medical record. The immunology drug allergy service successfully delabelled him to amoxicillin with a 3 day direct provocation challenge under medical supervision. As shown by $\mathrm{Li}$, et al. delabelling such patients to be treated with first line antibiotics which often are penicillins, reduce length of stay, and reduce the risk of multi-resistant organisms [9].

\section{Conclusion}

MIRM is a condition that occurs in children and less commonly in young adults that has a favourable prognosis. It is a distinct entity from SJS/TEN and EM. Clinicians should be aware of its features comprising inflammation at multiple mucosal sites with sparse or absent cutaneous lesions and investigate for M. pneumoniae infection. Caution needs to be exercised in not ascribing a drug hypersensitivity, especially a penicillin allergy and refer to an immunology service for further evaluation.

\section{References}

1. Gordon O, Oster Y, Michael-Gayego A, Marans RS, Averbuch D, et al. (2019) The Clinical Presentation of Pediatric Mycoplasma pneumoniae Infections-A Single Center Cohort. Pediatr Infect Dis J 38: 698-705. [Cossref]

2. Zão I, Ribeiro F, Rocha V, Neto P, Matias C, et al. (2018) Mycoplasma pneumoniaeassociated Mucositis: A Recently Described Entity. Eur J Case Rep Intern Med 5 : 000977. [Crossref] 
3. Li K, Harber RM (2012) Stevens-Johnson Syndrome Without Skin Lesions (Fuchs Syndrome): A Literature Review of Adult Cases With Mycoplasma Cause. Arch Dermatol 148: 963-964.

4. Amode R, Ingen-Housz-Oro S, Ortonne N, Bounfour T, Pereyre S, et al. (2018) Clinical and histologic features of Mycoplasma pneumoniae-related erythema multiforme: A single-center series of 33 cases compared with 100 cases induced by other causes. $J \mathrm{Am}$ Acad Dermatol 79: 110-117. [Crossref]

5. Canavan TN, Mathes EF, Frieden I, Shinkai K (2015) Mycoplasma pneumoniaeinduced rash and mucositis as a syndrome distinct from Stevens-Johnson syndrome and erythema multiforme: a systematic review. J Am Acad Dermatol 72: 239-245.

6. Li T, Lee N (2018) Mycoplasma pneumoniae-Associated Mucositis. N Engl J Med 379: 1262.
7. Simecka JW, Ross SE, Cassell GH, Davis JK (1993) Interactions of mycoplasmas with B cells: antibody production and nonspecific effects. Clin Infect Dis 17 Suppl 1: S176-S182. [Crossref]

8. Sassolas B, Haddad C, Mockenhaupt M, Dunant A, Liss Y, et al. (2010) ALDEN, an algorithm for assessment of drug causality in Stevens-Johnson Syndrome and toxic epidermal necrolysis: comparison with case-control analysis. Clin Pharmacol Ther 88: 60-68. [Crossref]

9. Li J, Shahabi-Sirjani A, Figtree M, Hoyle P, Fernando SL, et al. (2019) Safety of direct drug provocation testing in adults with penicillin allergy and association with health and economic benefits. Ann Allergy Asthma Immunol 123: 468-475.

Copyright: (C2020 Pillay R. This is an open-access article distributed under the terms of the Creative Commons Attribution License, which permits unrestricted use, distribution, and reproduction in any medium, provided the original author and source are credited. 